\title{
Physiotherapists' knowledge and the implementation of COVID-19 infection prevention and control measures
}

\author{
Wesam Saleh A. Al Attar ${ }^{\mathrm{a}, \mathrm{b}, \mathrm{c}, *}$ and Mohamed A. Husain ${ }^{\mathrm{d}}$ \\ ${ }^{a}$ Department of Physiotherapy, College of Applied Medical Sciences, Umm Al Qura University, Makkah, \\ Saudi Arabia \\ ${ }^{\mathrm{b}}$ Discipline of Exercise and Sport Science, Faculty of Medicine and Health Sciences, The University of Sydney, \\ Sydney, Australia \\ ${ }^{\mathrm{c}}$ Department of Sport, Exercise and Health, Faculty of Medicine, University of Basel, Basel, Switzerland \\ ${ }^{\mathrm{d}}$ Department of Physiotherapy, College of Health and Sport Sciences, University of Bahrain, Manama, Bahrain
}

Received 17 January 2021

Accepted 18 March 2021

\begin{abstract}
.
BACKGROUND: Physiotherapists are required to recognize their role in managing patients with Coronavirus Disease-19 (COVID-19), and to adopt preventive measures to limit transmission of the disease.

OBJECTIVE: The aim of this study was to assess the perception, knowledge, and application of the preventive measures taken by physiotherapists in managing issues with confirmed or suspected patients suffering from COVID-19.

METHODS: A self-administered survey comprising 15 questions was divided into four sections related to precautions when interacting with patients with COVID-19: (1) knowledge of the physiotherapy role, (2) knowledge of preventive measures to limit transmission of the virus, (3) practicing these measures, and (4) managing patients with COVID-19.

RESULTS: A total of 456 physiotherapists from 139 countries participated in the study. Most physiotherapists were knowledgeable regarding their role in the management of COVID-19 patients $(M=94.3 \%$; SD $=15.4)$ and the management of potential COVID-19 patients $(M=84.5 \%$; $\mathrm{SD}=20.1)$. The rating of knowledge and practices of preventive measures to limit transmission of COVID-19 were lower $(M=74.3 \%$; $\mathrm{SD}=25.7$, and $M=62.5 \%$; $\mathrm{SD}=31.3$, respectively). Participants from the European region $(M=83 ; \mathrm{SD}=15.8)$ had a higher score than participants from the Asia Western Pacific region $(M=78$; $\mathrm{SD}=18.49 ; P=0.01)$.

CONCLUSIONS: Physiotherapists are highly knowledgeable about their role in managing COVID-19 patients. Most of them are adopting preventive measures to limit the transmission of the disease. Yet, physiotherapists are required to enroll in medical education, training and infection control workshops and courses to remain updated with the recent advances in such fields.
\end{abstract}

Keywords: Health risk, viral transmission, disease control, infectious disease, SARS-CoV-2

\footnotetext{
*Address for correspondence: Wesam Saleh A. Al Attar, PT, MSc, PhD, Assistant Professor/Consultant of Physiotherapy, Department of Physiotherapy, College of Applied Medical Sciences, Umm Al Qura University, Makkah, Saudi Arabia. Mail address: PO Box: 715, Makkah, 21955, Saudi Arabia. Tel.: +966
}

54820 6504; E-mail: wsattar@uqu.edu.sa; ORCID: 0000-00031907-4539, twitter:@WSAAlAttar (Wesam Saleh A. Al Attar); ORCID: 0000-0002-2724-828X, twitter:@drmuraghi (Mohamed A. Husain). 


\section{Introduction}

The purpose of physiotherapy in managing hospital admitted patients with confirmed or suspected COVID-19 includes screening, referral, and treatment [1]. Physiotherapy management may also include techniques aimed at rehabilitating the musculoskeletal, neurological, and cardiopulmonary systems, resulting in coughing and expectoration of mucus [1]. In particular, cardiorespiratory physiotherapists are among the healthcare professionals supporting the management and care of these patient populations and always play a vital role in the non-invasive treatment such as postural positioning, mobilization during the period of weaning from invasive mechanical ventilator support [2]. Following ventilator support, physiotherapists are involved with respiratory muscle training, improving vital capacity, and promoting physical activity [3].

Consequently, physiotherapists must be careful when treating these patients since evidence indicates that non-invasive ventilation may increase the virus's risk of aerial spreading [1, 4]. However, patients infected with COVID-19 may show prognostic factors requiring management with invasive ventilation [5]. To emphasize the role of physiotherapists in treating such cases, Thomas et al. [1] published a clinical practice recommendation for physiotherapy management for COVID-19 in the acute hospital setting. The recommendations include outlines to assist the physiotherapy workforce in planning and responding to the increased demand for services and determining eligible patients with confirmed or suspected COVID-19 who should be treated by physiotherapists. Therefore, physiotherapists need to be aware of medical and cardiorespiratory management and infection transmission of patients with COVID-19 [1]. COVID-19 can be transmitted from an infected individual to a healthy person during the incubation period, which is between 2 to 14 days (about 2 weeks), and yet may not exhibit symptoms during that period [6]. Thus, early diagnosis is critical.

Public health and infection control measures are urgently needed to decrease the financial and medical burdens of COVID-19 and limit the global spread of the virus. Based on epidemiological experience gained from the SARS-CoV-2, history of travel, isolation of patients, and limiting human-tohuman interactions are effective methods to limit the virus's spread [7]. Also, the WHO recommends infection control interventions and advice for the public to reduce the general risk of transmission.
The recommendations include avoiding close contact with people suffering from acute respiratory infections, unprotected contact with farm or wild animals, and frequent hand-washing, especially after direct contact with patients or their environment [8]. When the risk of infection is high such as being in an area where the COVID-19 has started to spread or traveling from an area where it is spreading, guidance should be sought from the WHO, healthcare provider, and national public health authority for accurate information $[8,9]$. The purpose of this study was to assess physiotherapists' perception of their role in managing issues related to the COVID-19 disease from case identification, limiting transmission in different clinical settings, and treating patients with mild symptoms.

\section{Subjects and methods}

\subsection{Survey development}

To the authors' knowledge, no validated surveys are addressing the perception and knowledge of physiotherapists in managing issues associated with COVID-19. Therefore, a survey was developed specifically for the current study.

Broad questions were adapted from the current interim guidance and information for healthcare workers published by the WHO, World Physiotherapy (WPT), and the Centers for Disease Control and Prevention (CDC) [10-12]. Physiotherapy-specific questions were constructed based on information published on open-access sources of information such as Physiopedia and WPT [11, 13]. Lastly, respiratory Physiotherapy related questions were adapted from the position paper of the Italian Association of Respiratory Physiotherapists (ARIR) [2]. Pilot testing was performed before the beginning of the study to assess each question's content validity. The survey was distributed to thirty physiotherapists from different WPT members organizations, deemed representative of the final testing cohort due to their varying experiences. The physiotherapists were from diverse subspecialties and different native languages.

Each question was rated for clarity, comprehension, and appropriateness on a scale of 1.0 to 5.0 (lowest to highest). Questions scoring an average of fewer than 4.0 points in any parameter were considered as 'weak' in terms of clarity, comprehension, and appropriateness, and so would be discarded. Nevertheless, all questions scored above 4.5 and were considered suitable for this study. 
The self-reported survey consisted of sociodemographic questions and 15 questions based on the knowledge of physiotherapists' infection control practices. The latter part of the survey aimed at collecting information related to the understanding of the role of the physical therapist in managing issues related to the COVID-19 disease, including case identification, limiting transmission in different clinical settings, and treating patients with mild symptoms.

Respondents were prompted to answer every question provided in the survey before the next set of questions were presented. A respondent should answer a maximum of 21 questions categorized into three sections: (1) the study invitation and participation agreement, (2) demographic questions, and (3) infection control practice and knowledge. The 15 questions comprising the survey were designed to explore the role of physiotherapists in four domains: knowledge of Physiotherapy role in the management of COVID-19 patients (3 questions); knowledge of preventive measures limiting the transmission of COVID-19 (5 questions); practicing preventive measures to limit transmission of COVID-19 (3 questions); and management of potential COVID-19 patient (4 questions). See Appendix A. Items were in the form of multiple-choice questions, and each item's scoring was dichotomous (correct answer $=1$; incorrect answer $=0$ ). The survey's maximum score is the sum of all items (a score of 15) and transformed into a percentage score for ease of data analysis and data reporting. Similarly, the scores for the survey domains were transformed into percentages. When questions are answered correctly, higher scores reflect more knowledge and perception (range of correct answers $0-15$ ). The respondents were able to see the total score, correct answers, and the questions they missed immediately after submission.

\subsection{Survey dissemination}

Global associations who are members of the WPT confederation were contacted, identifying potential participants to achieve a convenient physiotherapist sample. The participants were registered members of their representative associations under the umbrella of the WPT confederation. The invitation provided a brief background to the survey and encouraged physiotherapists with or without experience in managing issues associated with the COVID-19 to participate. Interested respondents clicked on an electronic link that led them to the survey description. Once the participants read the survey's scope and aim, they were asked to provide informed consent and access the survey. The survey was filled anonymously and electronically via a web-based form. Responses were collected from April through June 2020.

\subsection{Sample size and statistical analysis}

There are 625,000 physiotherapy members in the WPT confederation around the world [14]. Considering a 5\% margin of error at a $95 \%$ confidence level, the target number of physiotherapists was 384 [15]. To achieve the target number of physiotherapists, all associations registered in the WPT were contacted to distribute the survey among their members.

Responses were organized in Microsoft Excel 365 (Microsoft Corporation, Redmond, Washington, USA) and analyzed using Statistical Package for the Social Sciences (SPSS) version 24.0 (IBM Corp., Armonk, New York, USA). Descriptive statistics were computed to describe the sample characteristics and survey data. One-way Welch ANOVA was used to determine differences in the overall survey score based on respondents' qualifications and WPT regions. Tamhane's T2 post hoc test was used to explore pairwise comparisons.

\subsection{Study registration and ethics}

The current study is registered at the WPT in the COVID Physio Registry [16]. The Biomedical Ethics Committee at Umm Al Qura University reviewed and approved the study. Approval No. (HAPO02K012202010463). All participants in this study provided consent to take part in the study.

\section{Results}

\subsection{Participants}

A total of 456 physiotherapists from 139 countries worldwide participated in the study achieving $119 \%$ of the desired sample size. Of the total sample, about $53.3 \%(n=243)$ were female, $70.4 \%(n=321)$ were between 20 to 29 years of age, and $41 \%(n=188)$ were master's degree holders. Most participants (94.7\%, $n=432$ ) worked in the healthcare system. Participants' information is described in Table 1.

\subsection{Descriptive statistics of the survey data}

The average overall survey scores varied from $13.3 \%$ to $100 \%$ for the total sample, with an average 
of 78.6\%. United Arab Emirates (100.0\%), Canada (93.0\%), Australia (89.0\%), and Switzerland (96.0\%) were among the countries that scored the highest. In contrast, the overall scores of Madagascar (40.0\%),

Table 1

Participant information

\begin{tabular}{lc}
\hline Gender & No. $(\%)$ \\
Male & $213(46.7)$ \\
Female & $243(53.3)$ \\
Age group & \\
20-29 years & $321(70.4)$ \\
30-39 years & $111(24.3)$ \\
40-49 years & $19(4.2)$ \\
50-60 years & $5(1.1)$ \\
Highest qualification & \\
Diploma or equivalents & $4(0.9)$ \\
Bachelor of Science (BSc) or equivalent & $158(34.6)$ \\
Master of Science (MSc) or equivalent & $188(41.2)$ \\
Doctor of Physical Therapy (DPT) or equivalent & $67(14.7)$ \\
Doctor of Philosophy (Ph.D.) or equivalent & $39(8.6)$ \\
Workplace & \\
Healthcare system & $432(94.7)$ \\
Academia & $24(5.3)$ \\
WPT region & \\
Africa region & $47(10.3)$ \\
Asia Western Pacific region & $180(39.5)$ \\
European region & $132(28.9)$ \\
North America Caribbean region & $71(15.6)$ \\
South America region & $26(5.7)$ \\
\hline
\end{tabular}

Abbreviations: WPT, World Physiotherapy
Russia (40.0\%), and Myanmar (20.0\%) were among the lowest. The average score (percentage) per country is shown in Fig. 1.

For the domain-specific scores, the highest average score for the total sample was for the knowledge of physiotherapy role in the management of COVID-19 patients $(M=94.3$; $\mathrm{SD}=15.4)$ as well as the management of potential COVID-19 patients ( $M=84.5$; $\mathrm{SD}=20.1$ ). In contrast, the domains of knowledge and practices of preventive measures to limit transmission of COVID-19 were lower than the other two domains $(M=74.3 ; \mathrm{SD}=25.7$, and $M=62.5$; $\mathrm{SD}=31.3$ ) (Table 2).

Regarding the number of participants per country, the highest participation rates were from Saudi Arabia $(n=82)$, the United States of America (USA) $(n=55)$, Australia, and the United Kingdom (UK) $(n=19$ each $)$.

\subsection{Differences in the overall score based on participant's qualifications and WPT region}

There was a statistically significant difference in the overall score based on participants' qualifications $\left(\mathrm{F}_{2,244.7}=35.1, \quad P<.001\right)$. Post-hoc comparisons revealed no differences in the overall score between
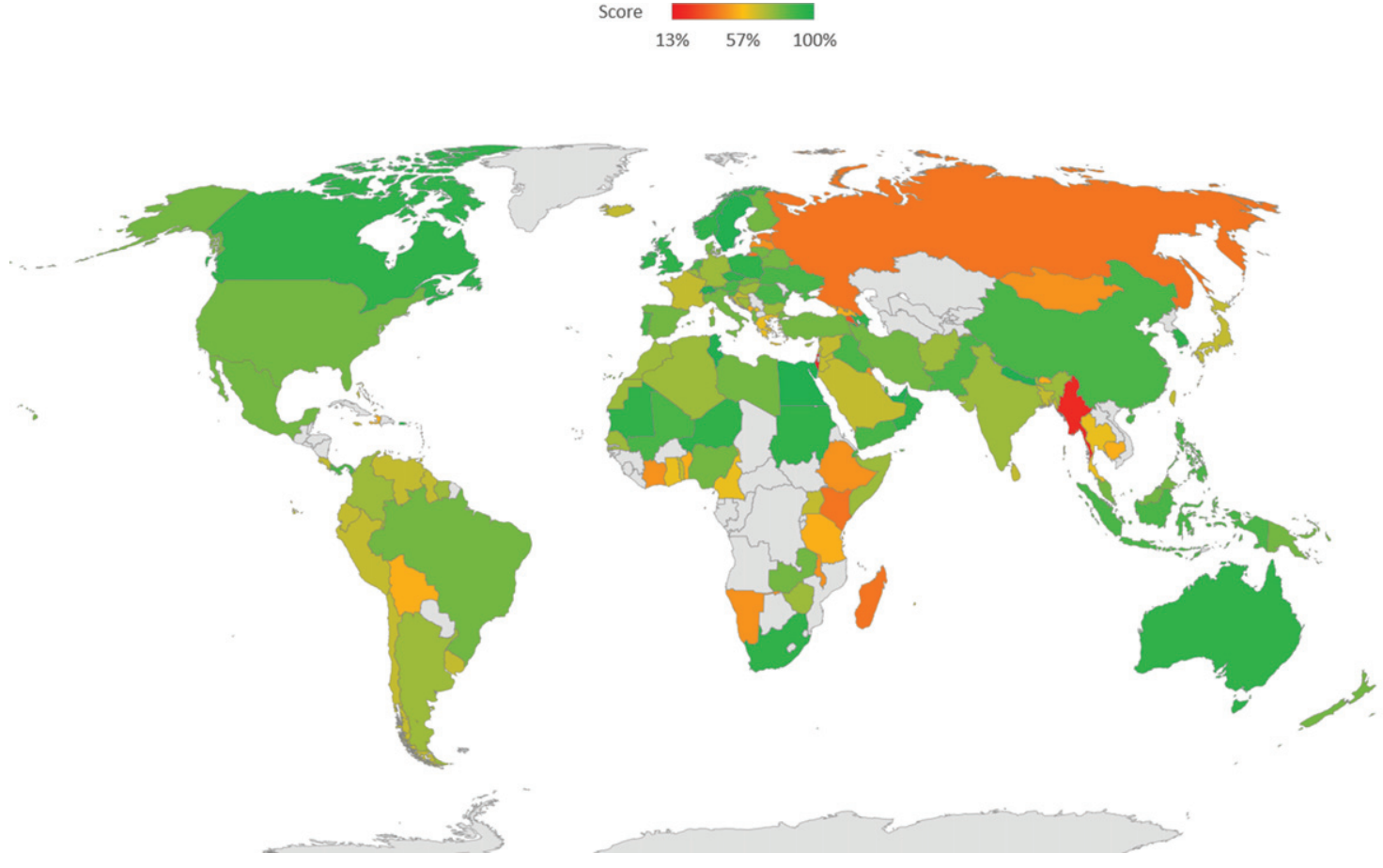

Fig. 1. Survey score distribution by country. 
Table 2

Domain-specific item analysis

\begin{tabular}{lcc}
\hline & $\begin{array}{c}\text { Correct } \\
\text { answers } \\
\text { No. (\%) }\end{array}$ & $\begin{array}{c}\text { Incorrect } \\
\text { answers } \\
\text { No. (\%) }\end{array}$ \\
\hline Knowledge of physiotherapy roles in the management of COVID-19 patients & & \\
$\quad$ Caring about respiratory issues & $433(95)$ & $23(5)$ \\
$\quad$ Caring for overall functioning & $428(94)$ & $28(6)$ \\
Perception of telemedicine as an option of service delivery & $428(94)$ & $28(6)$ \\
Knowledge of preventive measures limiting transmission of COVID-19 & & \\
Physical distance guidelines & $368(81)$ & $88(19)$ \\
Understanding the concept of contact & $336(74)$ & $120(26)$ \\
Proper hygiene & $411(90)$ & $45(10)$ \\
Knowledge of major COVID-19 symptoms & $375(82)$ & $81(18)$ \\
$\quad$ Wearing personal protective equipment & $203(45)$ & $253(55)$ \\
Practicing preventive measures to limit transmission of COVID-19 & & \\
$\quad$ Reducing the risk of spreading infection among health workers & $396(87)$ & $60(13)$ \\
Following protocol to limit the spread of infection at the workplace & $251(55)$ & $205(45)$ \\
Promoting Proper Hygiene in the workplace & $208(46)$ & $248(54)$ \\
Management of potential COVID-19 patients & & \\
$\quad$ COVID-19 history taking & $348(76)$ & $108(24)$ \\
Advising family members to limit the spread of infection & $318(70)$ & $138(30)$ \\
Advising COVID-19 patients to limit the spread of infection & $428(94)$ & $28(6)$ \\
Advising a COVID-19 patient to stay in a well-ventilated room & $446(98)$ & $10(2)$ \\
\hline
\end{tabular}

participants with doctoral degrees $(M=83.4 ; \mathrm{SD}=$ 20.0) and masters' degrees $(M=83.5 ; \mathrm{SD}=14.5$; $P>0.05)$. However, participants with doctoral degrees $(M=83.4 ; \mathrm{SD}=20.0)$ and masters' degrees $(M=83.5 ; \mathrm{SD}=14.5)$ had higher overall scores compared to participants with bachelors' degrees $(M=69.8 ; \mathrm{SD}=17.02 ; P<0.001)$.

Results also showed a significant difference in the overall score based on the participant's region of the WPT $\left(\mathrm{F}_{4,125.97}=3.48 ; P=0.01\right)$. Post-hoc comparisons revealed that participants from the European region $(M=83 ; \mathrm{SD}=15.8)$ had a higher score than participants from the Asia Western Pacific region $(M=78 ; \mathrm{SD}=18.5 ; P=0.01)$. All other pairwise comparisons between WPT regions were statistically not significant $(P>0.05)$.

\section{Discussion}

We set out this cross-sectional survey study to assess physiotherapists' knowledge and practice regarding their role in managing issues related to the COVID-19 pandemic. Our findings showed that, on average, physiotherapists are highly knowledgeable about their role in managing COVID-19 patients. However, despite their knowledge regarding preventive measures that limit COVID-19 transmission, their practice of such preventive measures was inadequate.
To the best of our knowledge, there is no other investigation of the perception and knowledge of physiotherapists in managing issues associated with the COVID-19. Physiotherapy can help manage patients with COVID-19 who have respiratory problems, physical problems, or both [1]. Physiotherapy might be indicated for patients with COVID-19 who present with copious airway secretions that they cannot clear independently. The role of physiotherapists in the management of respiratory conditions, including COVID-19, include monitoring and addressing respiratory fatigue, preventing prolonged immobility complications, and assisting in the recovery of the patient's autonomy in activities of daily life, which are often compromised by prolonged periods of sedation and prolonged hospitalization [2] Also, in the ICU, physiotherapy services can be instrumental as physiotherapists may provide airway clearance techniques for ventilated patients and assist in positioning patients with severe respiratory failure associated with COVID-19 [1]. Most of our study participants were aware of all these roles.

The Italian Association of Physiotherapy, in collaboration with the professional registry for physiotherapists, has drafted an essential document for operational suggestions in conjunction with the COVID-19 pandemic to arise [2]. This document included dealing with all non-essential treatments through telerehabilitation modality and managing clinical cases via telephone or webcam counseling. 
More than $90 \%$ of the respondents of the current study were mindful of such service delivery options. Telemedicine is an initiative designed to reduce contact between people and avoid the spread of contagions provoked by patients' travels and contact with other people infected with COVID-19. Additionally, it is a crucial choice from the economic point, particularly for private sectors since many physiotherapists can ensure continuity of treatment through telerehabilitation.

The level of knowledge about COVID-19 preventive measures among the current study participants is considered high. However, there is a percentage of participants who lack such knowledge, particularly concerning wearing personal protective equipment (PPE). The WHO indicated a priority for research to focus on actions that can save lives and protect health care workers and the community from transmission to create a safe working environment. These actions include optimizing the use of protective equipment and other infection prevention and control measures in both health care facilities and community settings [1, 17, 18]. Thomas et al. [1] summarized the recommendations for physiotherapy respiratory interventions, including cough etiquette, aerosol-generating procedures, sputum induction, mobilization, and exercise. These recommendations are vital for the physiotherapy workforce who are involved with treating patients suffering from COVID-19.

The latest statistics released by the WHO show a rapidly increasing number of confirmed COVID19 cases. Europe and the Americas reported the highest number of cases [19]. In the current study, Northern America was represented with a noticeable participation rate from the USA and Canada. They also showed a prominent level of awareness of COVID-19 infection prevention and control measures. Similarly, participants from the European region also showed an overall moderate to elevated levels of awareness. In contrast, the Western Pacific Region (A) countries, which consist of Australia, Brunei, Japan, New Zealand, and Singapore, reported the lowest numbers of reported cases but were among the leading countries in awareness level. New Zealand followed an elimination strategy, like China, which involved border management, disease surveillance, public communication, research, and evaluation [20]. Such strategies seem to reduce the number of cases compared to some European and Northern American countries, which followed a suppression strategy involving total lockdowns and intense physical distance. However, the latter strategy may have failed to contain the disease. Thus, it appears that the national strategy in handling the COVID-19 crisis does not only affect the number of cases, but it exceeds that to include the level of awareness in handling such cases.

In a study from Vietnam, $88.4 \%$ of the healthcare workers showed the right level of knowledge about COVID-19 as opposed to our study participants at $78.6 \%$ [21]. Considering the two studies' differences regarding the surveys, the participants of the current study correctly recognized the COVID-19 symptoms (82\%) compared to the Vietnamese study (72.8\%). Another study showed that $79.6 \%$ of physicians know the symptoms of COVID 19, and $85.4 \%$ of doctors know about hand hygiene, social distancing, and PPE [22].

The ongoing pandemic nature of COVID-19 made it necessary for healthcare workers to adopt increased precautions and put effort into implementing appropriate hygienic conditions and following recommendations [21, 22]. In contrast to our results showing inadequate preventive measures limiting COVID-19 transmission, a study from Pakistan study showed good practice of such measures [23].

Two previously published studies correlated the educational level with the knowledge and perception of outbreaks $[24,25]$. The current study showed a significant and positive association between participants' qualifications, knowledge about COVID-19, and its protective measures and practices. This finding agrees with a previously published study from China during the COVID-19 pandemic [26].

The study has two limitations that can be addressed in future research. First, the survey data did not include the sub-specialties or the workplace of the physiotherapists who participated in the study. Thus, it is not possible to determine if those who work in hospital settings are familiar with the COVID-19 preventive and control measures or not. Second, the lack of similar studies made it challenging to compare this study's results and other similar work. Nevertheless, the authors do not believe that these limitations affected the conclusions reached.

\section{Conclusions}

The COVID-19 outbreak brings great challenges to healthcare systems. Many healthcare providers from various departments, including physiotherapy, who might have some clinical experience in infection 
care, are included in managing this novel pandemic. Therefore, training and education are needed [27]. Also, continuous medical education and training are recommended to ensure that medical teams are adequately prepared to deal with public health emergencies [28]. It is considered the first study to address the COVID-19 knowledge, perception, and practice among physiotherapists to the best of our knowledge.

\section{Acknowledgments}

The authors acknowledge Prof. Ross H. Sanders, Prof. Oliver Faude, Prof. Mohammed Alghamdi, Dr. Ahmed Qasem. Dr. Fahad Alkabkabi, Dr. Ibrahim Alramadhani, Dr. Nasser Alshamrani and Dr. Hend A. Dorgham, for writing assistance, technical editing, language editing, and proofreading of the manuscript. The authors would like to thank all physiotherapists who participated in this study.

\section{Conflict of interest}

The authors declare that they have no conflicts of interest relevant to the contents of this review.

\section{Ethical statement}

This project was reviewed and ethically approved by the Biomedical Ethics Committee at Umm Al Qura University. Approval No. HAPO02K012202010463. Consent was obtained from all participants.

\section{Funding}

The authors have not declared a specific grant for this research from any funding agency in the public, commercial or not-for-profit sectors.

\section{References}

[1] Thomas P, et al. Physiotherapy management for COVID-19 in the acute hospital setting: clinical practice recommendations. Journal of Physiotherapy. 2020;66(2):73-82. doi: 10.1016/j.jphys.2020.03.011.

[2] Lazzeri M. et al. Respiratory physiotherapy in patients with COVID-19 infection in acute setting: a Position Paper of the Italian Association of Respiratory Physiotherapists (ARIR). Monaldi Arch Chest Dis. 2020;90(1), doi: 10.4081/monaldi.2020.1285.
[3] Felten-Barentsz KM, et al. Recommendations for HospitalBased Physical Therapists Managing Patients With COVID19. Physical Therapy. 2020;100(9):1444-57, doi: 10.1093/ ptj/pzaa114.

[4] Seto W, et al. Effectiveness of precautions against droplets and contact in prevention of nosocomial transmission of severe acute respiratory syndrome (SARS). The Lancet. 2003;361(9368):1519-20, doi: 10.1016/S0140-6736(03) 13168-6.

[5] Ding L, Wang L, Ma W, He H. Efficacy and safety of early prone positioning combined with HFNC or NIV in moderate to severe ARDS: a multi-center prospective cohort study. Crit Care. 2020;24(1):28, doi: 10.1186/s13054-020-2738-5.

[6] Petersen E, et al. Comparing SARS-CoV-2 with SARS-CoV and influenza pandemics. The Lancet Infectious Diseases, pp. S1473309920304849, Jul. 2020, doi: 10.1016/S14733099(20)30484-9.

[7] Kim JY, et al. The First Case of 2019 Novel Coronavirus Pneumonia Imported into Korea from Wuhan, China: Implication for Infection Prevention and Control Measures. J Korean Med Sci. 2020;35(5):e61, doi: 10.3346/jkms.2020. 35.e61.

[8] World Health Organization, Coronavirus disease (COVID19) advice for the public, World Health Organization, Jun. 04, 2020. https://www.who.int/emergencies/diseases/ novel-coronavirus-2019/advice-for-public (accessed Jul. 08, 2020).

[9] World Health Organization, Coronavirus disease (COVID19) Situation Report - 162, World Health Organization, Jun. 2020. Accessed: Jun. 07, 2020. [Online]. Available: https:// www.who.int/docs/default-source/coronaviruse/20200 630-covid-19-sitrep-162.pdf?sfvrsn=e00a5466_2.

[10] World Health Organization, Coronavirus disease (COVID19) pandemic, World Health Organization, Jul. 07, 2020. https://www.who.int/emergencies/diseases/novel-corona virus-2019 (accessed Jul. 08, 2020).

[11] World Physiotherapy, COVID-19 Information Hub, World Physiotherapy, 2020. http://world.physio/resources/covid19-information-hub (accessed Jul. 08, 2020).

[12] CDC, Coronavirus Disease 2019 (COVID-19), Centers for Disease Control and Prevention, Feb. 11, 2020. https://www. cdc.gov/coronavirus/2019-ncov/index.html (accessed Jul. 08, 2020).

[13] Physiopedia contributors, Role of the Physiotherapist in COVID-19, May 28, 2020. https://www.physio-pedia.com/ Role_of_the_Physiotherapist_in_COVID-19 (accessed Jul. $08,2020)$.

[14] World Confederation for Physical Therapy, About us, 2020. https://world.physio/about-us (accessed Jul. 10, 2020).

[15] Maple Tech International LLC, Sample Size Calculator, Calculator.net, 2020. https://www.calculator.net/samplesize-calculator.html type $=1 \& \mathrm{cl}=95 \& \mathrm{ci}=5 \& \mathrm{pp}=50 \& \mathrm{ps}=62$ $5000 \& \mathrm{x}=67 \& \mathrm{y}=21$ (accessed Jul. 10, 2020).

[16] World Physiotherapy, COVID-19: COVIDPhysio Registry, World Physiotherapy, 2020. http://world.physio/covid-19information-hub/covid-19-covidphysio-registry (accessed Jul. 08, 2020).

[17] Sadeghi R, Heshmati H. Innovative methods in teaching college health education course: A systematic review. J Educ Health Promot. 2019;8:103. doi: 10.4103/jehp.jehp_357_18.

[18] Chu DK, et al. Physical distancing, face masks, and eye protection to prevent person-to-person transmission of SARS-CoV-2 and COVID-19: a systematic review and meta-analysis. The Lancet. 2020;395(10242):1973-87, doi: $10.1016 /$ S0140-6736(20)31142-9. 
[19] World Health Organization, WHO Coronavirus Disease (COVID-19) Dashboard, World Health Organization, Jun. 04, 2020. https://covid19. who.int/ (accessed Oct. 27, 2020).

[20] Baker MG, Kvalsvig A, Verrall AJ. New Zealands COVID19 elimination strategy. Med J Aust, Aug. 2020, doi: 10.5694/mja2.50735.

[21] Huynh G, Nguyen T, Tran V, Vo K, Vo V, Pham L. Knowledge and attitude toward COVID-19 among healthcare workers at District 2 Hospital, Ho Chi Minh City. Asian Pac J Trop Med. 2020;13(6):260-65, doi: 10.4103/19957645.280396.

[22] Bhagavathula AS, Aldhaleei WA, Rahmani J, Mahabadi MA, Bandari DK. Novel Coronavirus (COVID-19) Knowledge and Perceptions: A Survey of Healthcare Workers, Infectious Diseases (except HIV/AIDS), preprint, Mar. 2020. doi: 10.1101/2020.03.09.20033381.

[23] Saqlain M, et al. Knowledge, attitude, practice and perceived barriers among healthcare workers regarding COVID-19: a cross-sectional survey from Pakistan. Journal of Hospital Infection. 2020;105(3):419-23, doi: 10.1016/j.jhin. 2020.05.007.
[24] Alqahtani A, et al. Australian Hajj pilgrims knowledge, attitude and perception about Ebola, November 2014 to February 2015. Eurosurveillance. 2015;20(12):21072, doi: 10.2807/1560-7917.ES2015.20.12.21072.

[25] Holakouie-Naieni K, et al. Assessing the Knowledge, Attitudes, and Practices of Students Regarding Ebola Virus Disease Outbreak. Iran J Public Health. 2015;44(12): 1670-6.

[26] Zhong B.-L, et al. Knowledge, attitudes, and practices towards COVID-19 among Chinese residents during the rapid rise period of the COVID-19 outbreak: a quick online cross-sectional survey. Int J Biol Sci. 2020;16(10):1745-52, doi: 10.7150/ijbs.45221.

[27] Speroni KG, Seibert DJ, Mallinson RK. Nurses Perceptions on Ebola Care in the United States, Part 2: A Qualitative Analysis. JONA: The Journal of Nursing Administration. 2015;45(11):544-50, doi: 10.1097/NNA.00000000000 00261.

[28] Li L, Xv Q, Yan J. COVID-19: the need for continuous medical education and training. The Lancet Respiratory Medicine. 2020;8(4):e23, doi: 10.1016/S2213-2600(20)30125-9. 